\title{
La masificación de la educación y la búsqueda de igualdad, justicia y equidad sociales en Colombia'
}

\author{
Mass education and the pursuit of social equality, \\ justice and equity in Colombia
}

Faustino Peña Rodríguez ${ }^{2}$

Resumen

Con la implementación de la masificación educativa, referirse a la importancia de la educación por parte de la sociedad, los organismos internacionales, la normatividad, los planes nacionales de desarrollo y los planes decenales, se convirtió en un asunto recurrente. Sin embargo, su desarrollo no ha logrado disminuir las desigualdades, inequidades e injusticias sociales. El Estado colombiano la ha afrontado con políticas públicas marcadas por el vaivén y la improvisación, lo cual ha dificultado el objetivo de la igualdad, justicia y equidad sociales.

\section{Palabras clave}

Masificación educativa, igualdad, equidad, justicia, desarrollo.

\section{Abstract}

With the implementation of mass education, reference to the importance of education by society, international agencies, regulations, national development plans, and decennial plans has become a recurrent issue of discussion. However, its development has failed to decrease inequalities, inequities and social injustices. The Colombian State has been faced with public policies marked by instability and improvisation, which have made it difficult to achieve the goal of social equality, justice and equity.

Key words:

Mass education, equality, equity, justice, development.

Artículo recibido el 25 de marzo de 2012 y aprobado el 19 de julio de 2012.

1 Artículo inscrito en el marco de la tesis doctoral Distribución social del capital escolar en Colombia, Universidad Pedagógica Nacional, Universidad del Valle y Universidad Distrital.

2 Profesor-investigador Universidad Pedagógica Nacional, Bogotá, Colombia.

Correo electrónico: fprodriguez@pedagogica.edu.co 
A la educación se le nombra de diferentes maneras y para diferentes procesos: como eje fundamental para la construcción de una sociedad cohesionada, democrática y abierta (Informe de la Misión de Sabios -en adelante Misión, 1996-; Delors, 1996); para asegurar el progreso social y cultural del país y romper el círculo vicioso de la pobreza y la exclusión (Misión, 1996; Delors, 1996; Tedesco, 2000); para formar ciudadanos libres y creativos, autónomos e innovadores (Misión, 1996); para formar los científicos y técnicos que posibiliten acelerar el desarrollo económico y social que el país requiere (Misión, 1996; Plan Decenal de Educación -PDE- 1996-2005; PDE 2006-2015; Ley 29 de 1990; Ley 1286 de 2009); como factor de equidad social (Tedesco, 2000; Gómez, 1999). Se señala que el cambio no es posible sin educación (PDE 1996-2005) y se le asigna la finalidad de construir bienes públicos (Gómez, 1999). Los Planes Nacionales de Desarrollo -PND- del período 1978-2006 abordan la problemática de la equidad, igualdad y justicia sociales desde justificaciones y conceptualizaciones que varían entre uno y otro gobierno. Asimismo, actualizan el discurso a los planteamientos y movimientos que se están dando en el plano internacional.

En las últimas décadas la educación ha estado asociada a desarrollo, en cuanto se considera que a través suyo se puede lograr el despliegue completo del hombre en sus diferentes facetas y dimensiones. Sin embargo, a pesar de los discursos y las propuestas, las desigualdades sociales no ceden y las políticas planteadas para enfrentarlas tienen efectos perversos. La ampliación de la cobertura educativa desarrollada en las últimas décadas contrasta con la poca movilidad social, equidad e igualdad sociales y se afinca la percepción de que el Estado colombiano tiene tendencia a invertir más en los que tienen más (Gómez, 1999), lo que conduce a cuestionar el sistema educativo en particular y el sistema social en general por sus imperfecciones.

\section{Educación y sociedad}

Las relaciones entre educación y sociedad han sido consideradas importantes y, por tanto, objeto de análisis por parte del Estado, las sociedades, los individuos y los organismos internacionales, hecho que produce que esta realidad esté atravesada por múltiples perspectivas de estudio.

En la década de los 90 del siglo XX, el Estado colombiano conformó una nueva misión con la finalidad de analizar las relaciones entre ciencia, educación y desarrollo. A diferencia de las que se conformaron al comienzo de la segunda mitad del siglo, esta misión estuvo conformada por científicos colombianos, quienes se dieron a la tarea de pensar estas relaciones y, con ello, el país. El título del informe producido al respecto es diciente: Colombia al filo de la oportunidad, en el cual se sustenta que "la educación es el eje fundamental para la construcción de una sociedad cohesionada sobre la base de una ética que promueva la tolerancia, la solidaridad, la participación democrática, la equidad y la creatividad" (Misión, 1996, p. 12).

La Misión considera que la educación es un factor fundamental para mejorar la calidad de vida de los colombianos y que la creatividad se puede liberar a través de las ciencias y las artes, asegurando el progreso social y cultural del país. El problema radica en cambiar concepciones y percepciones de la sociedad sobre la educación, sus finalidades y logros: "Nuestra sociedad demanda un sistema educativo capaz de formar ciudadanos libres y creativos, autónomos e innovadores, sin quienes no será posible consolidar una sociedad democrática y abierta, inserta en la economía global y en la cultura contemporánea" (Misión, 1996, p. 27).

La Misión propuso un nuevo programa de educación llamado Cosmología, que abarca 12 años -un año de preescolar, nueve de educación básica, dos de educación media-, el cual se basa en enseñar a pensar conceptualmente de acuerdo con el conocimiento global, hecho fundamental para que Colombia desarrolle la educación, la ciencia y la tecnología durante el siglo XXI. Se necesita llevar a cabo un programa de formación y capacitación capaz de formar científicos y técnicos para acelerar el desarrollo económico y social. En esta perspectiva, considera que la primera década del siglo XXI será una de las más importantes en la historia del país, y para ello se requiere consolidar los centros de in- 
vestigación existentes, iniciar 1600 nuevos grupos de investigación y 60 nuevos institutos de excelencia (Misión, 1996).

También se presenta cierto consenso en el sentido que la educación tiene como finalidad la construcción de bienes públicos; esto es, bienes mediante los cuales se obtienen beneficios comunes: "Lo público es una inmensa abstracción, es una enorme generalización y se necesita un dominio elevado de la razón, de la lógica, del pensar causal, de la abstracción simbólica, para manejarlo" (Villaveces, 1999, p. 57). La pregunta por el genuino interés del público conduce al cuestionamiento que formula Gómez (1999, p. xxvi): ¿formación de punta para unos pocos, o formación masiva pero mediocre? Esto implica que el carácter de bien público de la educación no debe esconder su cualidad como bien privado que la gente convierte en capital, con el que mercadea en el plano social procurando ganancias y dividendos.

En materia pública ¿hacia dónde deben apuntar las acciones del Estado? Gómez (1999) considera que deben dirigirse hacia tres objetivos: educar a los más pobres, costear los bienes que la iniciativa privada no produciría por sí sola y asegurar información transparente acerca de las diferentes opciones educativas. En su calidad de pública, la educación debe ser accesible a toda la población en edad escolar, de todos los niveles (Ocampo, 2000).

Dada la problemática social y cultural de Colombia, se presenta la siguiente proyección como reto para el país:

En el próximo siglo, además de mejorar la calidad de la educación actual y fortalecerla como instrumento para construir una patria más amable, Colombia tendrá que hacer un esfuerzo mucho mayor que el de los noventa -que no fue despreciable- para aumentar la cantidad y la equidad de su educación para todos los colombianos. Un proceso amplio de reforma y consolidación institucional que catapulte un nuevo modelo horizontal de educación -base real de la equidad-, podría contribuir a ese propósito que cada vez más necesitaremos los colombianos en el nuevo siglo del conocimiento (Londoño, 2000, p. 84).

\section{Educación y sociedad en la perspectiva de las políticas internacionales}

Desde la segunda mitad del siglo XX nacen y se multiplican las atribuciones formuladas a la educación por parte de la Unesco, mediante las cuales se asocia a su carácter público (1950), su calidad de derecho fundamental de todos (Coombs, 1968), al desarrollo (1990), como elemento esencial para que los individuos fructifiquen sus talentos y capacidades (1990), base para el establecimiento de una democracia auténtica y para la competitividad internacional (Unesco, 1962; Cepal-Unesco, 1992), su carácter de tesoro individual (1996) y como elemento indispensable para la consecución de paz, libertad y justicia social (1996).

En sus primeras acepciones, el desarrollo se plantea como el elemento a través del cual se consigue el despliegue completo del hombre en sus diferentes facetas y dimensiones - postulado del informe “Aprender a ser" (Faure, 1972) acogido por Delors, 1996-. Escobar (1996) plantea que los organismos internacionales conceptúan el desarrollo como el proceso progresivo, ordenado y estable mediante el cual se transita de una situación a otra, se pasa de relaciones arcaicas a la modernización, para lo cual la industrialización y urbanización se constituyen en rutas progresivas e inevitables hacia la mundialización. El desarrollo se instauró como modelo de igualdad, equilibrio y de democracia social, buscaba que los ciudadanos tuvieran igual acceso a los bienes y servicios que la modernidad y la modernización propugnaban (Martínez, Noguera y Castro, 2003).

En el informe Delors (1996) se concibe la educación al servicio del desarrollo económico y social $\mathrm{y}$ del fortalecimiento de un nuevo humanismo. El objetivo de esta relación es conseguir mayor equidad social a partir de la transformación de las estructuras productivas.

Los propios organismos que implementaron el desarrollo se encargaron de realizar su evaluación con miras a profundizarlo; Cepal-Unesco (1992) consideraron que los sistemas educativos de capacitación y de ciencia y tecnología habían crecido, pero presentaban insuficiencias en la calidad de sus resultados, en su pertinencia respecto a los re- 
querimientos sociales y económicos y al grado de equidad con el que accedían los diferentes estratos sociales. Al mismo tiempo, plantearon que a finales de los setenta y comienzos de los ochenta se agudizó la pobreza y aumentó la exclusión social.

Slater -citado por Escobar, 1996- plantea que un rasgo esencial del desarrollo es la voluntad de poder espacial, la cual queda explícita en expresiones como primer y tercer mundo, norte y sur, centro y periferia, de tal manera que a partir de estos términos se producen diferencias, subjetividades y órdenes sociales. El desarrollo se presenta como una manera de gobernar, de hacer que los pueblos sean gobernables, en tanto se requiere que existan elementos articuladores de la implementación de las políticas y las acciones de los Estados. Los organismos internacionales vieron en la educación la forma de llegar a las poblaciones con discursos y prácticas más o menos homogéneos, que les posibilitaran adelantar los proyectos asociados al desarrollo: "Una de las ideas centrales de los procesos de transformación educativa ha sido la hipótesis según la cual la educación es un factor de equidad social. La prioridad a la educación en las estrategias de desarrollo estuvo siempre basada en el argumento según el cual ella es la única variable que afecta simultáneamente la equidad social, la competitividad económica y el desempeño ciudadano" (Tedesco, 2000, p. 6).

Contrario al planteamiento que tradicionalmente se formula, en el sentido que una educación exitosa genera equidad social, Tedesco (2000) pregunta cuánta equidad social es necesaria para que haya una educación exitosa. Al respecto plantea:

El objetivo de lograr mayor equidad social a través de la educación, no depende sólo de cambios en la oferta pedagógica. La equidad es un fenómeno sistémico y, por lo tanto, sin modificaciones sustanciales en los patrones de distribución del ingreso será muy difícil avanzar en los logros educativos que permitan a la población tener acceso a niveles de educación adecuados para su incorporación productiva a la sociedad. (Tedesco, 2000, p. 8)

La equidad, igualdad y justicia son fenómenos sociales, lo cual significa que la educación se cons- tituye en una de las variables a examinar, pero no es la única.

\section{Desarrollo económico y desigualdad social}

Los discursos y prácticas en torno al desarrollo y sus bondades, sus relaciones con crecimiento económico y al papel que desempeña la educación para que ello sea posible, se han vuelto una constante en la política colombiana -al igual que en la latinoamericana- desde la segunda mitad del siglo XX. Esto conduce a la pregunta sobre la forma en que la tríada desarrollo, crecimiento económico y educación ha coadyuvado a la disminución de las desigualdades sociales, pues está basada en un discurso que no logra ubicar concretamente al individuo, sujeto o estrato social específico a la que va dirigida.

Recursos humanos y desarrollo son dos temas muy vinculados entre sí. El reconocimiento de esta vinculación ha inducido a la Cepal a iniciar [...] un esfuerzo sistemático para profundizar en las interrelaciones entre el sistema educativo, la capacitación, la investigación y el desarrollo tecnológico en el marco de los elementos centrales de su propuesta, es decir, la transformación productiva, la equidad social y la democratización política. (Cepal-Unesco, 1992, p. 15)

Estos discursos chocan con las inconsistencias del Estado al incrementar el gasto social en educación, el cual efectivamente aumentó la cobertura pero no la inversión, aumento que se dio en detrimento de algunas instituciones públicas, tal como se evidencia en el siguiente informe:

\footnotetext{
Mientras durante la mayor parte de los años noventa el gasto social creció de manera sustancial, pasando de representar el 8,5\% del PIB en 1991 al 13\% en 1999, los recursos destinados a la educación superior aumentaron sólo el 0,21\% puntos porcentuales entre 1990 y 1995 y 0,16\% puntos entre 1995 y 1999, mientras que la matrícula oficial en educación superior se incrementó 9,3\% en el primer período y $36 \%$ en el segundo. (Universidad Nacional, 2007, p. 29)
}

La propuesta formulada por Cepal-Unesco en 1992 señala que la transformación productiva con equidad significa crecimiento económico 
con equidad, lo cual conlleva un esfuerzo interno imprescindible, indelegable e impostergable de los países; competitividad y equidad en un contexto de sustentabilidad ambiental; recursos humanos y difusión del progreso técnico; readecuación del Estado y apoyo externo para posibilitar la transformación productiva con equidad, aspecto que comparte el Informe Delors: “[...] el crecimiento económico a ultranza no se puede considerar ya el camino más fácil hacia la conciliación del progreso material y la equidad, el respeto de la condición humana y del capital natural que debemos transmitir en buenas condiciones a las generaciones futuras" (Delors, 1996, p. 15).

Una de las dificultades para hacer posible la equidad surge cuando las oportunidades de acceso se dan a través de políticas educativas direccionadas desde el subsidio y el crédito, incluso en los primeros niveles escolares, como lo evidenciaron las palabras del expresidente Samper al presentar al país el Informe de la Misión de Sabios: "En la búsqueda de la equidad, facilitaremos, por medio del otorgamiento de subsidios y de créditos, el acceso y permanencia de estudiantes de escasos recursos a la Educación Básica Primaria" (Misión, 1996, p. 12). Política que evidencia que el problema no es de falta de recursos sino de distribución: "En el futuro, el problema de la distribución puede llegar a ser más agudo que el de la pobreza. Si bien los indicadores tendenciales de la pobreza muestran diferencias notables entre grandes ciudades, pequeños centros urbanos y población dispersa, en general la pobreza disminuye en el mediano y largo plazo. No pasa lo mismo con la desigualdad" (Sarmiento, 1999, p. 184).

El presidente del período 1990-1994 hace referencia, no a desarrollo humano, sino a capital humano: "La formación del capital humano es no sólo la clave del futuro porque implica una mayor inversión en la gente sino porque es un camino a la equidad" (Misión, 1996, p. 42). Esta forma de ver el desarrollo y, específicamente, el desarrollo humano, da lugar a políticas y acciones que justifican las inequidades y desigualdades como producto de recursos económicos limitados.
Los sistemas educativos no pueden responder de manera indefinida a una demanda que crece constante y fuertemente. Tendrían que ofrecer al mismo tiempo las mismas posibilidades de educación a todo el mundo, respetar la diversidad de gustos y de culturas y satisfacer todos los tipos de demandas. Dadas las limitaciones financieras, es obligatorio asignar los recursos en la mejor forma posible a fin de conciliar cantidad y pertinencia, equidad y calidad. A falta de un modelo único de distribución óptima, la atribución de los recursos debe, sobre todo, reflejar claramente las decisiones colectivas correspondientes a los arbitrajes que cada sociedad efectúa para asegurar su desarrollo económico, social y cultural (Delors, 1996, p. 180).

De igual manera, justifica que el Estado, apoyado o condicionado por organismos internacionales, priorice sus inversiones en los niveles que en su criterio le resultan más eficientes y eficaces desde la perspectiva económica. Este lenguaje de los mayores rendimientos termina siendo discriminador, en tanto tradicionalmente no se han construido políticas robustas que ataquen los problemas de raíz (alfabetización, cobertura de la educación básica, apoyo a los sectores más discriminados), lo que produce que cada gobierno vuelva sobre lo mismo de diferente manera -como lo evidencia el análisis de los Planes Nacionales de Desarrollo PND, ítem 4-. La posible salida es entonces conciliar educación, desarrollo y crecimiento económico:

La educación ha recuperado su importancia estratégica en la discusión del desarrollo económico. Más allá de la concepción del capital humano de los sesenta, se ha encontrado como un determinante crítico del hoy denominado "crecimiento endógeno". Así, el diferente ritmo de expansión económica de los países se debe en buena medida a su capacidad de innovar y a la velocidad con la cual el cambio tecnológico es asimilado por la sociedad; y estas dos características están estrechamente asociadas con la cantidad y calidad de educación existente en cada país. La educación no es solamente un gasto social que el Estado hace en los más pobres, sino, ante todo, una inversión socialmente rentable y una garantía de acceso 
equitativo. Es pues un elemento estratégico para el desarrollo económico sostenido". (Sarmiento, 1999, p. 185)

\section{Equidad, igualdad y justicia sociales en la normatividad educativa}

El perfeccionamiento y desarrollo social del estudiante se encuentran en el centro del espíritu del Decreto 080/1980. En sus líneas, la educación superior no podrá estar limitada por consideraciones de raza, credo, sexo o condición económica o social. Serán las capacidades académicas las que direccionen el acceso a la educación superior en el marco de igualdad de oportunidades. Este Decreto plantea como objetivos de la educación superior (artículo 22) impartir educación para la realización plena del colombiano, lo cual posibilite la configuración de una sociedad más justa, equilibrada, autónoma, que se vincule dignamente a la comunidad internacional; igualmente, ampliar las oportunidades de acceso a la educación superior propiciando, a través de programas específicos, la incorporación de estudiantes de las zonas urbanas, rurales e indígenas marginadas del desarrollo económico y social, para que alcancen un desarrollo vital en su propio contexto. De hecho, la preocupación por la eficiencia del sistema educativo es una pretensión de vieja data:

La Constitución Nacional, en su artículo 41, consagra el derecho a la educación primaria gratuita y obligatoria para todos los colombianos y le asigna al Estado la obligación de protegerla, fomentarla y cualificarla. El Estado reglamenta la promoción automática, al considerar que mejora sensiblemente la eficiencia del sistema educativo por disminuir las tasas de remitentes y de deserción, potencializando la universalización de la educación primaria. (Decreto 1469 de 1987)

La Ley 30 de 1992 plantea como objetivos de la educación superior y sus instituciones promover la unidad nacional, la descentralización, la integración regional y la cooperación interinstitucional, con la finalidad de que las diversas zonas del país dispongan de los recursos humanos y de las tecnologías que les permitan atender adecuadamente sus nece- sidades. En sus artículos 111-116, hace referencia a facilitar el ingreso a la educación superior a las personas de escasos ingresos económicos y ordena a la nación, entes territoriales y a las propias instituciones, establecer una política general de becas, ayudas y créditos para estos estudiantes. La educación superior será accesible a quienes demuestren las capacidades requeridas y cumplan con las condiciones académicas exigidas en cada caso (Ley 30 de 1992).

La Ley que regula la educación en general plantea que "la educación es un proceso de formación permanente, personal, cultural y social que se fundamenta en una concepción integral de la persona humana, de su dignidad, de sus derechos y sus deberes. (Ley 115 de 1994, artículo 1). En consecuencia, corresponde al Estado, a la sociedad y a la familia velar por la calidad de la educación y promover el acceso al servicio público educativo, $y$ es responsabilidad de la nación y de las entidades territoriales, garantizar su cubrimiento (Ley 115 de 1994, artículo 4). Por su parte, el Decreto 1860 de 1994 (artículo 4) plantea que "todos los residentes en el país sin discriminación alguna, recibirán como mínimo un año de educación preescolar y nueve años de educación básica que se podrán cursar directamente en establecimientos educativos de carácter estatal, privado, comunitario, cooperativo, solidario o sin ánimo de lucro".

Como producto de lo estipulado en la Constitución Política Nacional, en 1996 se elaboró el primer Plan Decenal de Educación -PDE- 1996-2005. Estos planes -consonantes con las políticas internacionales- parten del principio que "la necesidad de dotar al país de un Plan Nacional de Desarrollo Educativo se fundamenta en la comprensión de que la educación, como principal fuente de saber, se constituye en la época actual en la más cierta posibilidad de desarrollo humano, cultural, económico y social de la nación" (PDE 1996-2005, p. 10). Se señala allí que ningún cambio es posible sin educación, pues su significado y valor proviene de los objetivos sociales y culturales que el país define y su importancia para llevar a cabo el proyecto de 
nación, así como desarrollar los aspectos económicos y políticos de la sociedad.

Cuando finalizaba el siglo XX, uno de tantos diagnósticos sobre la situación socioeconómica del país señaló la inequitativa distribución del ingreso, elevadas tasas de pobreza, diversas manifestaciones de violencia política, delincuencia común, inseguridad e intolerancia ciudadanas (PDE 1996-2005). El primer Plan Decenal de Educación evidenció que la búsqueda en el país de la equidad, justicia social, superación de las desigualdades de acceso y permanencia en el sistema educativo, y acceso de los grupos más pobres y poblaciones de las zonas rurales a los niveles secundario y de educación superior, eran aún objetivos por cumplir:

Nuestra sociedad registra aún profundas inequidades. Los más pobres no están recibiendo las mismas oportunidades de calidad y cobertura del servicio educativo que los sectores medio y alto. Existen diferencias sensibles entre la educación que se ofrece en las ciudades principales y en las zonas rurales. Aún es necesario trabajar mucho para disminuir la diferencia de oportunidades atribuibles a factores étnicos o de género. (PDE 1996-2005, p. 15)

La interrelación de los beneficios sociales e individuales de la educación debe conducir a los derechos básicos propios de una sociedad democrática y moderna (empleo, seguridad social, participación política, acceso a servicios culturales, científicos y tecnológicos, mejora del nivel de ingreso económico), aspectos ligados a las oportunidades brindadas a individuos y grupos sociales de obtener mayores niveles de educación (PDE 1996-2005).

La búsqueda de igualdad y equidad vuelve a aparecer en el PDE 2006-2015, en el cual se consigna que al Estado corresponde garantizarlas a toda la población, desde la educación inicial hasta su articulación con la educación superior:

La educación es un proceso de formación integral, pertinente y articulado con los contextos local, regional, nacional e internacional que desde la cultura, los saberes, la investigación, la ciencia, la tecnología y la producción, contribuye al justo
La masificación de la educación y la búsqueda de igualdad, justicia y equidad sociales en Colombia

Faustino Peña Rodríguez

desarrollo humano, sostenible y solidario, con el fin de mejorar la calidad de vida de los colombianos, y alcanzar la paz, la reconciliación y la superación de la pobreza y la exclusión. (PDE 2006-2015, p. 13)

Aunque los PDE son indicativos para los encargados de la política y la administración en los niveles nacional, departamental y municipal, evidencian las formas en que se ha constituido la nación y sus respectivas problemáticas. En estos planes hay referencia a una serie de poblaciones que debe cobijar la política educativa y que en un país con mayor justicia, equidad e igualdad sociales no sería necesario mencionar tan explícitamente:

Gestionar y asignar recursos de inversión para garantizar el acceso y la permanencia en la educación desde la primera infancia hasta el nivel superior, que incluya la población vulnerable, con necesidades educativas especiales, grupos y comunidades indígenas, afrocolombianos, raizales, rom, urbana marginal, rural dispersa, madres cabeza de familia, adultos, destinados al mejoramiento y al fortalecimiento de sus potencialidades; priorizando los recursos para construir equidad territorial, urbana, rural y social en general (PDE 2006-2015, p. 68).

Las leyes de ciencia y tecnología van a ofrecer un lenguaje diferente pero orientado a los mismos propósitos: el mejoramiento de la vida y la cultura del pueblo (Ley 29 de 1990). La Ley 1286 de 2009 señala que su objetivo es "fortalecer el Sistema Nacional de Ciencia y Tecnología y a Colciencias para lograr un modelo productivo sustentado en la ciencia, la tecnología y la innovación, para darle valor agregado a los productos y servicios de nuestra economía y propiciar el desarrollo productivo y una nueva industria nacional". Esta Ley sienta las bases para la consolidación de una política de Estado en ciencia, tecnología e innovación, como en su momento lo hizo el PDE 1996-2005 con la educación para evitar que estuviera sujeta al vaivén de los gobernantes, sin que en ninguno de los dos casos el objetivo se haya cumplido. 


\section{Equidad, igualdad y justicia sociales en los Planes Nacionales de Desarrollo 1978- 2006}

Los Planes Nacionales de Desarrollo -PND- del período 1978-2010, dan cuenta de la reiteración de las metas de la universalización de la educación primaria, en un principio, y luego de la educación básica durante nueve años, las cuales se presentan una y otra vez sin haberlas conseguido. Es característico de estos PND involucrar niveles escolares, programas o acciones sociales que le imprimen una impronta al Gobierno respectivo pero no necesariamente continúan unas ya iniciadas, con el agravante que esas originalidades no encuentran correspondencias entre unas y otras.

Los PND abordan la problemática de la equidad, igualdad y justicia sociales, desde variadas justificaciones y conceptualizaciones. Para el PND 1978-1982, el bajo desarrollo del país se debe al mal diseño de las políticas y los diagnósticos -no a la insuficiencia de recursos-, en cuanto no dirigen sus acciones al origen de las problemáticas, sumado a que el sector social no sabe manejar los recursos, lo que genera marginados y produce daños colaterales. Para mejorar la equidad en el acceso a la educación superior, este PND propone ampliar el sistema de crédito educativo y crear un fondo de bienestar que beneficie a los estudiantes de menores recursos.

Por su parte, el PND 1982-1986 consideró que para la sociedad resulta más gravoso excluir que incluir; presentó las inequidades sociales y regionales como producto de la concentración de las oportunidades educativas y laborales en unas pocas ciudades del país, y la poca pertinencia de la educación superior con las necesidades de las ciudades y regiones más pequeñas; considera que ha sido tradición que el Estado financie a los estratos altos y medios a través de matrículas bajas. Las alternativas propuestas para los sectores más desfavorecidos son la educación a distancia en los diferentes niveles escolares, así como el fortalecimiento del SENA.

El PND 1986-1990 considera que las inequidades se deben, en gran parte, a la ineficiencia interna del sector educativo; se plantea que el analfabetismo tiene su origen en la pobreza económica y el grado de desarrollo económico y social de las diferentes regiones del país. Crear una sociedad más justa requiere implementar un nuevo modelo de desarrollo económico que equilibre el desarrollo de las distintas regiones del país, consolidar líneas de crédito estudiantil, crear un fondo de becas dirigido a estudiantes pobres y redefinir el sistema de aportes públicos a la universidad.

El PND 1990-1994, a su vez, considera que las inequidades son el producto de no haber puesto suficiente cuidado en el fortalecimiento del capital humano, a la apertura de nuevas oportunidades y al desarrollo del país; para lograr estos aspectos es necesario hacer más equitativo y eficiente el sistema de financiación pública de la educación.

Para el PND 1994-1998, la equidad y la distribución del ingreso son posibles en la medida en que se ponga atención a la formación del capital humano y se involucre a los diferentes actores sociales en su consecución.

El PND 1998-2002 considera que la equidad es producto de expandir las oportunidades sociales y trabajar en las capacidades que la población requiere. Es la exclusión social la responsable de que el país no haya superado las inequidades y la pobreza.

El PND 2002-2006 plantea que las inequidades sociales son producto de la baja cobertura y calidad de la educación, lo que ha conducido a desigualdades por no haber aumentado la educación de los colombianos y haber concentrado las oportunidades en unos pocos.

El PND 2006-2010 considera que las inequidades son producto de la poca competitividad del país; para ser competitivos, es necesario trabajar en aspectos centrales como educación, investigación, aplicación de conocimientos, capital físico y humano, crédito popular y el apoyo al emprendimiento.

En resumen, las inequidades, desigualdades e injusticias sociales son interpretados en los PND de los períodos 1978-2006 como producto de:

- El diseño y diagnóstico de las políticas (19781982).

- El manejo de los recursos sociales (1978-1982, 1986-1990). 
- La tendencia del Estado a excluir (1982-1986; 1998-2002).

- La concentración de las oportunidades educativas y laborales en unas pocas personas y regiones del país (1982-1986; 1986-1990; 1990-1994; 1998-2002; 2002-2006).

- La financiación de la educación superior a los sectores de más altos recursos (1982-1986).

- No formar el capital humano (1990-1994; 1994-1998).

- La ineficiencia en la financiación de la educación pública (1990-1994).

- No involucrar los diferentes sectores sociales en el desarrollo del país (1994-1998).

- La baja cobertura y calidad de la educación (2002-2006).

- La poca competitividad del país (2006-2010).

La equidad, igualdad y justicia sociales en la sociedad colombiana han sido una búsqueda constante, que se incrementó con la masificación educativa. Una de las razones de la recurrencia discursiva y programática es la inequitativa distribución del ingreso, lo cual ha generado pobreza, discriminación, diferenciación social y cultural, exclusión y marginalidad, entre otras situaciones.

Todos los gobiernos han planteado esta situación en los Planes Nacionales de Desarrollo y han llevado a cabo acciones, tomando la educación como eje para superarla o disminuirla. Sin embargo, no se ha realizado a partir de una política de Estado que obligue y la aleje del vaivén de los diferentes sectores sociales y gobiernos.

En el plano educativo, la política está signada por bandazos donde la constante es su relación con el desarrollo económico y, por ello, ligada a situaciones variantes y discontinuas que imposibilitan políticas sostenibles en el corto, mediano y largo plazo.

\section{Las cifras de las desigualdades sociales y los esfuerzos para disminuirlas}

Datos de 1987 señalan que un 61,3\% de los admitidos a la universidad en ese año, provenía del $40 \%$ más rico de las familias y solo un 3,4\% provenía del 20\% más pobre (Gómez, 1999). La búsqueda de pertinencia en la educación superior ha ido en
La masificación de la educación y la búsqueda de igualdad, justicia y equidad sociales en Colombia

Faustino Peña Rodríguez

contra de las clases menos favorecidas y ha sido convertida en otra forma de eficacia, eficiencia y productividad, en tanto se dirigen los aprendizajes hacia lo necesario, lo cual es definido por el otro, mientras las élites siguen un proyecto sistemático. Es evidente que en Colombia las tradiciones producen tradiciones ligadas a privilegios, lo cual dificulta que se produzcan cambios sociales:

Sin excepción alguna, la probabilidad de alcanzar un nivel de escolaridad más avanzado es menor para las personas levantadas en hogares cuyo jefe fue menos educado. La asociación es más estrecha en los contextos más tradicionalistas, esto es, los hijos de padres no educados tienden a educarse menos en Guatemala que en Costa Rica, en Barranquilla que en Bogotá y en Ceará que en Sao Paulo. Vistas de otra manera, las cifras dicen que de 7 o más de cada 10 hijos de universitarios son universitarios, mientras 2 o menos de cada 10 hijos de personas con poca educación llegan a la universidad (las dos ciudades de Colombia son excepciones notables a este respecto). (Gómez, 1999, pp. 64-65)

En plena primera década del siglo XXI, en Colombia solo 35 de cada cien bachilleres acceden a la educación superior, se gradúan un poco más de la mitad de los que ingresan y deserta un gran porcentaje (Peña, 2012). Entre 1985 y 1997 la escolaridad del $10 \%$ más rico de la población económicamente activa subió dos años, la del 10\% más pobre subió en un grado. En este período, la diferencia de escolaridad entre el 10\% más rico de la población y el 10\% más pobre pasó de seis años en 1985 a siete años en 1993 (Sarmiento y otros, 2001).

Esta situación no se diferencia mucho de lo que ocurría, en décadas pasadas, en los demás niveles escolares y en el promedio de escolarización de la población colombiana. En 1988, la educación secundaria tenía una cobertura del 50\%, la cual, en su mayoría, era urbana, pues "solo tres de cada diez egresados rurales pasan a la Secundaria $\mathrm{Ru}$ ral" (OEI, 1996, p. 5). En el comienzo de la década de los ochenta, el promedio de escolaridad en Colombia fue de 3,8 años en el sector urbano y 
1,7 años en el rural; en 1988, 8,3 años en el sector urbano y 3,8 años en el rural (OEI, 1996).

La gravedad de estas cifras no es desconocida por el Estado, el cual, en muchas ocasiones, las afronta vía normatividad educativa y no por la de suplir las dificultades. Así, el acumulado de desigualdades sociales ronda niveles insospechados: “...según el Banco Mundial, en 1989 el ingreso promedio por habitante del $10 \%$ más rico de la población colombiana fue 37 veces el del 10\% más pobre, lo que dificulta la convivencia” (Misión, 1996, p. 101). La disparidad social entre los ricos y los excluidos dentro del país es enorme, lo cual obliga a la pregunta ¿para qué tanta inversión en educación si no es para lograr sociedades más justas y equitativas? Aun sosteniendo la importancia de la educación para el desarrollo humano y económico y formar capital humano, es evidente que esta no pretende la equidad, si se mira desde el gasto público: “[...] el gasto educacional ha perdido participación, en los últimos 25 años dentro del gasto social (de $42 \%$ en 1973 a $21,9 \%$ en 1995) y dentro del total del gasto del gobierno (de $13 \%$ en 1973 a 7,56\% en 1995). Esa pérdida se explica por el gasto de primaria y secundaria, puesto que el de la universidad permanece casi constante”. (Sarmiento, 1999, p. 187).

Esto evidencia un desajuste estructural pues, sin que el Estado haya conseguido la universalización de la educación básica y la media, se baja la inversión en estas y se mantiene en el nivel superior, desfavoreciendo a una gran cantidad y favoreciendo a unos pocos.

La Universidad Nacional (UN) estableció los Programas de Admisión Especial, bajo los cuales recibe estudiantes indígenas y a los mejores bachilleres de municipios pobres. Los datos de deserción de esta población son altos y preocupantes: un gran porcentaje perdió su calidad de estudiante en los tres primeros semestres (Bernal y Rincón, citados en UN, 2002), un $30 \%$ se retira sin finalizar estudios, cifra que no tiene diferencias notables con la de los estudiantes regulares; los indígenas exceden el promedio de permanencia dentro de la Universidad en un $44 \%$ frente a los estudiantes regulares, y los mejores bachilleres exceden el promedio de perma- nencia en un $30 \%$, cifra igual a la de los estudiantes regulares (Mayorga, 2002, citado en UN, 2007) sufriendo así el mayor efecto de superselección (Bourdieu y Passeron, 2001)'?

El estudio de la Universidad Nacional (2007) señala que la referencia a la deserción obliga a analizar la equidad y la igualdad, hecho que guarda relación con los bienes que poseen los individuos y grupos sociales, determinando su condición socioeconómica e incidiendo en sus condiciones de desempeño y trayectoria académica, con la forma en que el Estado trabaja para que las brechas disminuyan o aumenten. Esta investigación encontró, basándose en el año 2003, que hubo aumento de la cobertura de la educación superior, pero que no fue igualitaria ni equitativa en cuanto la cobertura bruta: para el quintil más rico de la población llegó al 73\%, mientras que para el más pobre llegó al 7\% (Universidad Nacional, 2007).

La poca movilidad, equidad e igualdad sociales echan al traste logros como la incorporación, en los últimos años, de población con menores cualidades académicas y de menores recursos económicos, lo que se ahonda con la deserción escolar universitaria, aumentando las desigualdades (OCDE, 1987; Santos, 1988; UN-Icfes, 2002; UN, 2007). La deserción produce que la cobertura real se vea seriamente afectada, y que los beneficios de la educación no sean directamente aprovechados en forma conjunta por los individuos, las instituciones y la sociedad, cuestionando el sistema educativo en su conjunto. En cuanto las deficiencias, son el producto de situaciones producidas y toleradas por este.

Producto de su mayor escolarización, una sociedad debe tener la capacidad de producir movilidad social en todos los que por ella pasan, y generar beneficios para el conjunto social, aspecto que se convierte en indicador de igualdad y equidad. Sin embargo, en Colombia la masificación escolar subió las oportunidades de prestigio a lugares a los

1 “Puesto que han debido superar una empresa de aculturación para satisfacer el mínimo indispensable de exigencias escolares en materia de lenguaje, los estudiantes de clases populares y medias que acceden a la enseñanza superior han sufrido necesariamente una selección más fuerte" (Bourdieu y Passeron, 2001, p. 91). 
que difícilmente pueden llegar los profesionales de los diferentes sectores sociales, reservándose las mejores para los sectores sociales dominantes. El aumento de escolarización se ha utilizado para que los mercados mantengan cierta estabilidad, al controlar los salarios y las oportunidades producto de un mayor número de profesionales, y no para impulsar la economía social y el desarrollo del país, por lo que a medida que avanza, en general, la escolarización, las condiciones sociales de los nuevos profesionales no se ven fortalecidas y sus disposiciones en este sentido así lo señalan.

\section{Referencias}

Bourdieu, P. y Passeron, J. (2001). La reproducción. Madrid: Editorial Popular.

Cepal, Unesco. (1992). Educación y conocimiento: eje de la transformación productiva con equidad. Santiago de Chile: Cepal.

Congreso de la República de Colombia. Ley 29 de 1990. Congreso de la República de Colombia. Ley 30 de 1992

Congreso de la República de Colombia. Ley 115 de 1994.

Congreso de la República de Colombia. Ley 1002 de 2005

Congreso de la República de Colombia. Ley 1286 de 2009.

Coombs, P. (1968). La crisis mundial de la educación. Barcelona: Península.

Delors, J. (1996). La educación encierra un tesoro. Comisión sobre la educación del siglo XXI. París: Unesco.

Departamento de Planeación Nacional. Plan Nacional de Desarrollo. Plan de Integración Nacional 1978-1982. Bogotá: Presidencia de la República.

Departamento de Planeación Nacional. Plan Nacional de Desarrollo. Cambio con equidad 1982-1986. Bogotá: Presidencia de la República.

Departamento de Planeación Nacional. Plan Nacional de Desarrollo. Plan de Economía Social 1986-1990. Bogotá: Presidencia de la República.

Departamento de Planeación Nacional. Plan Nacional de Desarrollo. La Revolución Pacífica 1990-1994. Bogotá: Presidencia de la República.

Departamento de Planeación Nacional. Plan Nacional de Desarrollo. El Salto Social 1994-1998. Bogotá: Presidencia de la República.
Departamento de Planeación Nacional. Plan Nacional de Desarrollo. Cambio para construir la paz 1998-2002. Bogotá: Presidencia de la República.

Departamento de Planeación Nacional. Plan Nacional de Desarrollo. Hacia un Estado Comunitario 2002-2006. Bogotá: Presidencia de la República.

Departamento de Planeación Nacional. Plan Nacional de Desarrollo. Estado Comunitario: desarrollo para todos 2006-2010. Bogotá: Presidencia de la República.

Escobar, A. (1996). La invención del Tercer Mundo. Construcción y deconstrucción del desarrollo. Bogotá: Norma.

Gómez, H. (Dir.). (1999). Educación: la agenda del siglo XXI. Hacia un desarrollo humano. Programa de Naciones Unidas para el Desarrollo. Bogotá: TM Editores.

Informe de la Misión de Sabios. (1996). Colombia al filo de la oportunidad. Bogotá: Presidencia de la República, Colciencias, TM Editores.

Londoño, J. (2000). Educación, desigualdad y crecimiento ¿qué aprender de América Latina? En Revista Educación y Cultura, 50, Bogotá: Fecode.

Martínez, A., Noguera, C., Castro, J. (2003). Currículo y modernización. Cuatro décadas de educación en Colombia. Bogotá: Cooperativa Editorial Magisterio.

Ministerio de Educación Nacional. (1976). Decreto 088. Bogotá: MEN.

Ministerio de Educación Nacional. (1980). Decreto 080. Bogotá: MEN.

Ministerio de Educación Nacional. (1987). Decreto 1469. Bogotá: MEN.

Ministerio de Educación Nacional. (1994). Decreto 1860. Bogotá: MEN.

Ministerio de Educación Nacional. Plan Decenal de Educación 1996-2005. Bogotá: MEN.

Ministerio de Educación Nacional. Plan Decenal de Educación 2006-2015. Bogotá: MEN.

Ocampo, J. (2000). Una educación para Colombia en el siglo XXI. En Revista Educación y Cultura, 50, 78-80. Bogotá: Fecode.

Organización de Estados Iberoamericanos -OEI-. (1996). Sistemas educativos nacionales (Colombia). Madrid: OEI.

Organización para la Cooperación y el Desarrollo Económico -OCDE-. (1987). Universities Under Scrutiny. París: OCDE. 
Peña, F. (2012). Distribución social del capital escolar en Colombia. Bogotá: Universidad Pedagógica Nacional, Universidad del Valle, Universidad Distrital, Mimeo.

Santos, B. (1988). De la idea de universidad a la universidad de ideas. En De la mano de Alicia: lo social y lo político en la postmodernidad, pp. 225-284. Bogotá: Siglo del Hombre Editores.

Sarmiento, A. (1999). Para una visión prospectiva de lo social: pobreza, equidad, educación y salud. En Gómez, H. (1999) ¿Para dónde va Colombia? Bogotá: TM Editores, Colciencias.

Sarmiento, A., Tovar, L. y Alam, C. (2001). Situación de la educación básica, media y superior en Colombia. Bogotá: Casa Editorial El Tiempo, Fundación Corona y Fundación Antonio Restrepo Barco.

Tedesco, J. (2000). Los desafíos de las reformas educativas en América Latina. En Revista Pedagogía y Saberes, 14, 5-14. Bogotá: Universidad Pedagógica Nacional.

Unesco. (1962). Situación demográfica, económica, social y educativa en América Latina. Proyecto principal de educación. Santiago de Chile: Unesco.

Unesco. (1990). Conferencia mundial sobre educación para todos y marco de acción para satisfacer las necesidades básicas de aprendizaje. Nueva York: Unesco.

Universidad Nacional de Colombia (2007). Cuestión de supervivencia. Graduación, deserción y rezago en la Universidad Nacional de Colombia. Bogotá: UN.

Universidad Nacional de Colombia. Icfes. (2002). Estudio de la deserción estudiantil en la educación superior en Colombia. Documento sobre estado del arte. Bogotá: UN, Icfes.

Villaveces, J. (1999). Racionalidad pública vs. Racionalidad privada ¿una falsa antinomia? En Gómez, H. (1999) ¿Para dónde va Colombia? Bogotá: TM Editores, Colciencias. 